\title{
OF COSMIC BACKGROUND ANISOTROPIES
}

\author{
G.F. SMOOT \\ Lawrence Berkeley Laboratory, Space Sciences Laboratory, \\ Center for Particle Astrophysics, and Department of Physics, \\ University of California, Berkeley, USA 94720
}

\begin{abstract}
Observations of the Cosmic Microwave Background (CMB) Radiation have put the standard model of cosmology, the Big Bang, on firm footing and provide tests of various ideas of large scale structure formation. CMB observations now let us test the role of gravity and General Relativity in cosmology including the geometry, topology, and dynamics of the Universe. Foreground galactic emissions, dust thermal emission and emission from energetic electrons, provide a serious limit to observations. Nevertheless, observations may determine if the evolution of the Universe can be understood from fundamental physical principles.
\end{abstract}

\section{Introduction}

With the discovery of the cosmic microwave background radiation the Big Bang has emerged as the standard model of cosmology. It is the goal of cosmologists to explain the origin and evolution of the Universe leading to what we observe in the present and then to predict what will occur in the future. An important example is the formation of large scale structure such as galaxies, clusters of galaxies, and even larger structures or voids. A major issue is the role of gravity. Modern cosmology uses the concepts of General Relativity to tie together the structure of space-time to the distribution of matter and energy as well as its dynamics - e.g. expansion. More recently quantum gravity has been considered in a role involving the creation of the Universe and its interesting contents. On a smaller scales we believe that gravity holds together all the structure that we observe. We assume it holds together stars, planets, the solar system, the stars in galaxies, clusters of galaxies, and so on. It is natural to assume that these structures form by gravitational instability. The otherwise smooth early Universe has seeds - density variations in an otherwise uniform and homogeneous medium. 
Regions that are more dense than average attract the material around them which in turn attracts more material and so on until the over-density goes non-linear and a stable structure forms. Slightly less dense regions would lose material to the more dense medium surrounding them. The less dense regions would then evolve to voids.

Observations of large scale structure led Edward Harrison and Yakov Zeldovich to speculate in 1970 and 1972 respectively that a scale-invariant spectrum of primordial density perturbations would grow into the combination of large scale structures present today. As a result the scale-invariant spectrum is often called the Harrison-Zeldovich spectrum. They selected a scale-invariant primordial spectrum though the data actually indicated that the dependence only needed to be close to scale invariant - roughly within the physical scale to the one half power. At that time there was no known process for producing initial perturbations on any scale of astronomical interest so that scale-free and, particularly, scale-invariant were appropriate choices. Cosmologists now commonly consider two mechanisms that produce nearly scale-invariant spectra. These are inflation (quantum fluctuations expanded to astronomical scales by accelerating expansion) and spontaneous symmetry breaking (topological defect relics of a higher energy space vacuum state). Each of these has implications for the actual density fluctuation spectra.

Observations of the spectral and angular distributions of the CMB provide a most powerful means for investigating the early Universe. The observation that the CMB has to very high precision a blackbody spectrum not only constrains the possible thermal history of the Universe but also allows us to rule out many alternative cosmological models to the Big Bang. Figure 1 shows the current observations of the CMB spectrum.

The CMB's place as the relic radiation of the Big Bang makes CMB anisotropy observations a direct probe of the early Universe. The discovery of anisotropy by the COBE DMR (Smoot et al. 1992, Bennett et al. 1992, Wright et al. 1992, Kogut et al. 1992) marks the start of the epoch when the CMB observations began to fulfill their promise of revealing what was actually happening in the early Universe. It also inspired a veritable avalanche of activity in the field with nine groups now reporting anisotropy and new theoretical work appearing nearly every day. A consensus has formed on the observations to be made and the implications and understanding that can be derived from these observations. The goals of CMB anisotropy observation and theory include those shown in Table 1. 


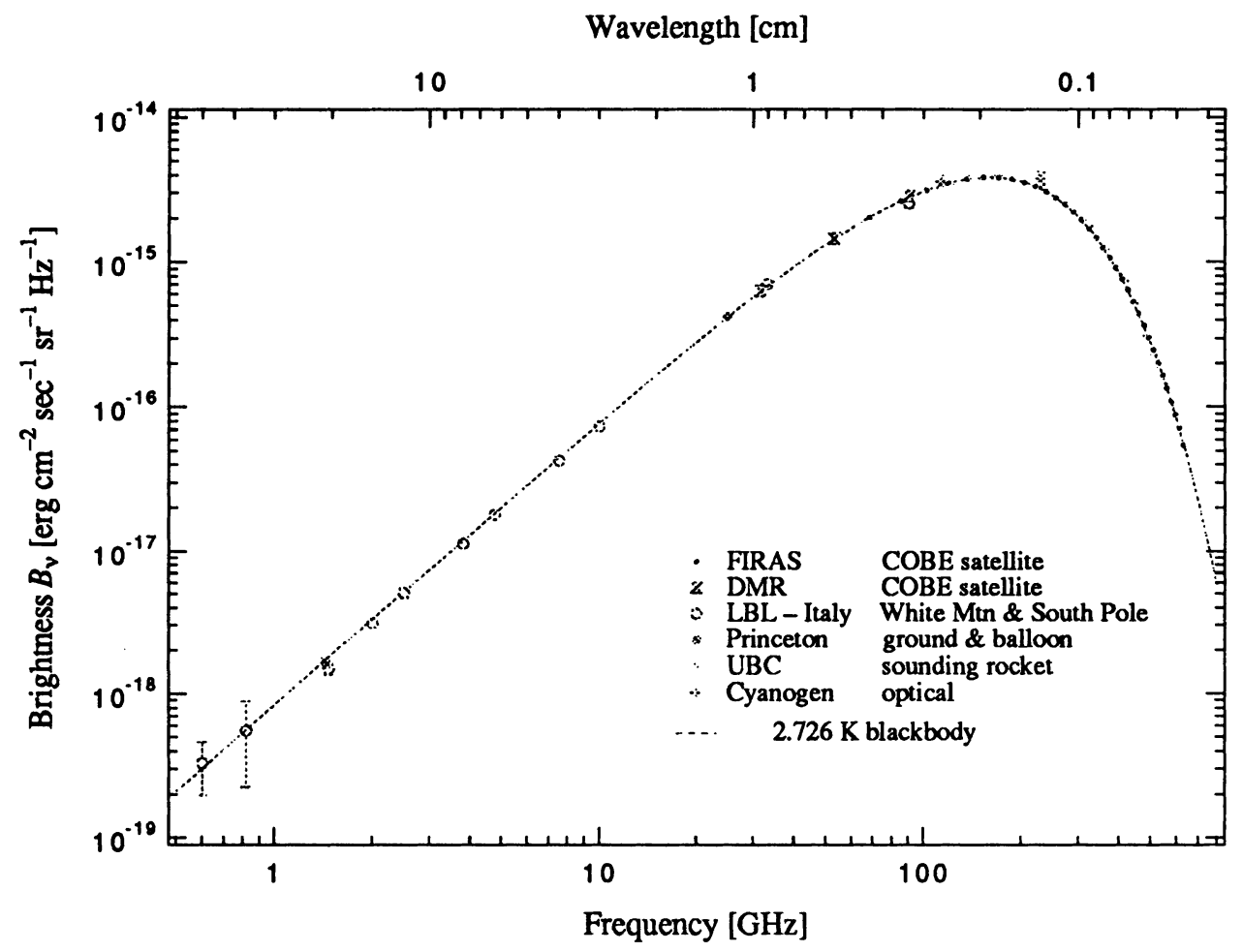

Figure 1. Current observations of the Cosmic Microwave Background spectrum: The measurements in the peak region are from COBE FIRAS (Mather et al. 1994) and the University of British Colombia rocket experiment (Gush et al. 1990) The Rayleigh-Jeans region data are from our LBL-Italy ground-based results from White Mountain and from the South Pole are shown together with a $2.73 \mathrm{~K}$ blackbody curve.

\section{COBE DMR Observations}

Measurements of anisotropy are clear and well-defined experimentally, even though technically difficult. One finds the sky brightness and brightness variations after calibrating the observations and removing the instrument signature and all foreground emissions. The results are interpreted in terms of our standard model. According to Big Bang theory the Universe is expanding from an original primeval fireball - a near thermal equilibrium state of very high density and temperature. As the Universe expanded it cooled, and when it reached a temperature of about $3000 \mathrm{~K}$ the primeval plasma coalesced to neutral atomic hydrogen and helium. At that time, about 300,000 years after the Big Bang, the cosmic background radiation was freed to move through the Universe with negligible scattering by free electrons. (This assumes a standard thermal history of the Universe, which implies no significant ionization of the Universe until redshifts less than 
TABLE 1. CMB Anisotropy Observation Goals

I Initial Conditions for Large Scale Structure Formation

The formation of galaxies, clusters of galaxies, and larger scale structures as well as voids is a key issue in cosmology.

\section{Physics of the Early Universe}

probes inflationary model predictions and/or quantum gravity.

test of topological defects - monopoles, strings, domain walls, textures.

probes the nature of the dark matter.

probes of the baryonic content of the Universe.

III Geometry and Dynamics of the Universe

directly traces metric of space-time and isotropy of expansion and space-time. probe the curvature of space through the detection \& location of Doppler peaks. measure the rotation and shear of the Universe.

about 100.) As a result the cosmic background photons are undisturbed, except for the universal expansion, since that epoch. If the primeval Universe was slightly inhomogeneous, the cosmic background radiation is slightly anisotropic.

The concept of a large angular scale anisotropy experiment is to look out from our local environment, past the solar system, past our Galaxy, past all galaxies and forming structure back to the cosmic photosphere. On large angular scales the dominant causes of anisotropy are gravitational redshift of the radiation (Sachs \& Wolfe 1967) and the motion of the observer (Doppler shift) which produces a dipole anisotropy.

The COBE Differential Microwave Radiometer (DMR) experiment was designed to map the microwave sky and find fluctuations of cosmological origin. For the $7^{\circ}$ angular scales observed by the DMR, structure is superhorizon (significantly larger than light could have traveled in the 300,000 years at last scattering) size so the features of the primordial perturbations are preserved unchanged since their primordial formation.

COBE was launched on 18 November 1989 into a $99^{\circ}$ inclination, terminator (day-night) polar orbit. The DMR instrument observes the difference in power received by two horn antennas each pointing $30^{\circ}$ from the space craft spin axis. The orbit and pointing of $C O B E$ result in a complete survey of the sky every six months while shielding the DMR from terrestial and solar radiation.

The DMR data were converted to maps that show the overall high level of uniformity of the CMB, the dipole anisotropy at about 1.2 parts per 
thousand (which is thought to be due to the Doppler effect caused by the motion of the solar system and Galaxy relative to the rest frame of the CMB), and fluctuations on all angular scales from the antenna resolution up to the quadrupole $\left(90^{\circ}\right)$ at levels of a few parts per million to about a part in 100,000 . A key question is: which part of the signal is due to fluctuations in the CMB relating to the last scattering surface and which part might be foreground effects? The DMR maps the sky at frequencies of $31.5,53$, and $90 \mathrm{GHz}$ (wavelengths of $9,5.7$, and $3.3 \mathrm{~mm}$ ). Multiple frequencies were used to help separate the cosmic signal from possible foreground sources, particularly Galactic emission.

\section{Separation of the Galactic and Cosmic Signals}

The DMR anisotropy maps are sufficiently sensitive and free from systematic errors that our knowledge of Galactic emission is a limiting factor in interpreting the measurements of the DMR maps (also eventually other observations). The detected signals are nearly constant when expressed in terms of antenna temperature. When converted to thermodynamic temperature based on variations of a $2.73 \mathrm{~K}$ Planckian spectrum, they are consistent with a single constant amplitude. On the other hand the antenna temperatures of the Galactic emissions: from synchrotron, free-free, and dust have strong frequency dependences. If approximated by a power law, the power law index is roughly: -2.75 to $-3.1,-2.1$, and $1.5 \pm 0.5$, respectively. The relative dependence and typical signal levels are shown in Figure 2.

Synchrotron emission arises when relativistic cosmic-ray electrons are accelerated by magnetic fields in the Galaxy. The energy spectrum of the cosmic ray electrons (a power-law with an index starting at about -2.75 and steepening with increasing energy) and the magnetic field strength determine the synchrotron emission effective power-law index. Free-free emission occurs when free electrons are accelerated by interactions with ions. Free-free emission is thermal bremsstrahlung radiation. It has nearly a flat intensity spectrum (index of -2 in antenna temperature) with a small modification accounted for by the Gaunt factor that brings the antenna temperature power-law index into the range -2.07 to -2.13 for reasonable interstellar plasma temperatures and densities.

The properties and factors that determine the emissivity of the dust at $\mathrm{cm}$ and $\mathrm{mm}$ wavelengths are a major topic of discussion. It is clear that dust is not an efficient radiator and absorber for wavelengths much larger than the size of the dust. Thus the antenna temperature of dust emission decreases at lower frequencies (larger wavelengths). The precise emissivity frequency dependence and temperature distribution of high galactic lati- 


\section{Galactic Foregrounds}

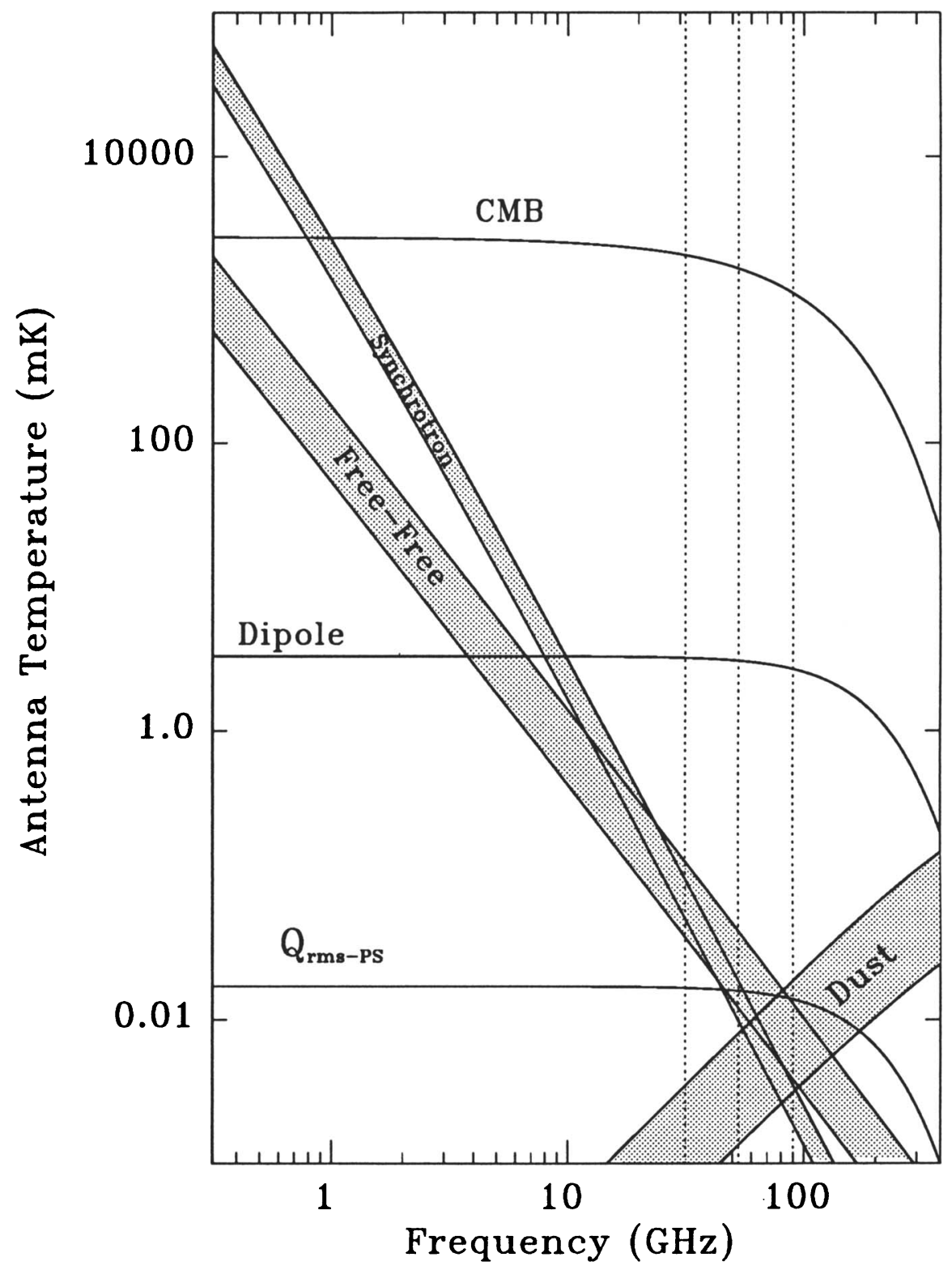

Figure 2. Galactic foregrounds compared with the Cosmic Microwave Background (CMB) signal all expressed in antenna temperature. (Antenna temperature is power per unit bandwidth per mode calibrated in Kelvin. It is defined by the relation: $P=$ $\mathrm{kT}_{a} \mathrm{~B}$, where $\mathrm{P}$ is the power, $\mathrm{k}$ is Boltzmann's constant, $\mathrm{T}_{a}$ is antenna temperature, and $B$ is the bandwidth.) The three vertical dotted lines indicate the COBE DMR observing frequencies. The width of the Galactic emission bands indicate the typical range of signal level for Galactic latitudes between $20^{\circ}$ and $70^{\circ}$. 
tude dust remain to be determined.

The flat spectral index of the DMR anisotropy, without correction for Galactic emissions, is consistent with a cosmic origin and inconsistent with an origin from a single Galactic component. Ganga et al.(1993) have shown that the 'MIT' FIRS balloon-borne bolometer observations correlate well with the COBE DMR maps. The FIRS bolometer observations have an effective frequency of about $170 \mathrm{GHz}$. The Tenerife (Hancock et al. 1994) ground-based observations of a strip in the northern sky have also been compared to a simulation of their observations on the COBE DMR maps indicating correlations (Lineweaver et al. 1994) and extending the spectral coverage down to $15 \mathrm{GHz}$ (2-cm wavelength). These additions make the flat spectral index argument stronger. However, from this fact alone we are unable to rule out a correlated superposition of dust, synchrotron, and free-free emission and thus more detailed Galactic emission models are required. The worst case scenario would be a dust component with an emissivity power law index that reached into the range 0 to about 1 in the $\mathrm{mm}$ wavelength range or pockets of very cold $(<4 \mathrm{~K})$ dust - either interstellar or cosmic. This seems an unlikely scenario but must be investigated carefully. Using maps made at non-DMR frequencies as well as the DMR maps, a multicomponent fit to CMB anisotropies and the three Galactic emissions indicates that the signal is predominantly CMB anisotropies (Bennett et al. 1992).

\section{COBE DMR Results}

We proceed assuming that the Galactic foreground emissions are small $(\leq 10 \%)$ compared to the CMB fluctuations. The DMR maps are a full-sky panorama showing a whole spectrum of primordial perturbations in the early Universe. Though some spots have statistical significance (e.g. Cayon \& Smoot 1994), in general the early DMR data signal to noise is roughly unity. To quantify the anisotropies, it is natural to expand the temperature fluctuations on the celestial sphere in spherical harmonics:

$$
T(\theta, \phi)=\sum_{\ell m} a_{\ell m} Y_{\ell m}(\theta, \phi)
$$

where $\ell$ is the Legendre polynomial number and $Y_{\ell m}$ are the spherical harmonics. A complete description of the CMB fluctuations would require observers to find the full set of coefficients $a_{\ell m}$. The DMR data can be fitted to determine the best estimated spherical harmonic coefficients.

At this stage we approach a somewhat simpler task: finding the mean power at each $\ell$. We are making use of the idea of rotational invariance of the mean power in the anisotropy. That is that there are no preferred 
directions. The direction independent mean square power at $\ell$ is $T_{\ell}^{2}=$ $\sum_{m} a_{\ell m}^{2}$. Since the COBE anisotropies are consistent with being drawn from a random-phase, Gaussian distribution (Smoot et al. 1994), then in principle the power spectrum contains the information characterizing the parent distribution.

We compare the power observed by the DMR to a scale-invariant (or Harrison-Zeldovich) spectrum by plotting the ratio as a function of $\ell$. Figure 3 shows a sample of the DMR measured anisotropy power spectrum. The data are clearly consistent with a scale-invariant (Harrison-Zeldovich) power spectrum. The question then becomes: what is the best estimate of the power spectrum and what limits can we place in an effort to exploit the CMB for learning cosmology as outlined in the table above? A careful analysis (Gorski et al. 1994) shows that the DMR data are very close to Harrison-Zeldovich with errors in power-law index of less than about 0.3 and that the amplitude normalized to the quadrupole $(\ell=2)$ is approximately $20 \mu \mathrm{K}$ or about $7 \times 10^{-6}$ of the CMB temperature. CMB anisotropy results are often ploted in terms of the mean power at some $\ell$ or band of $\ell$ 's normalized to a Harrison-Zeldovich spectrum (often as the equivalent quadrupole for this flat spectrum or as a dimensionless anisotropy).

The CMB power spectrum can readily be interpreted for what it tells us about the primordial power spectrum of fluctuations and then applied either backwards to tell us about early-Universe, high-energy physics or viewed forward in its relation to large scale structure. The field, especially theoretically and rightly so, is focused on these issues. However, there are other things that we can learn about the Universe using these data. We can learn a lot about the geometry and dynamics of the Universe and set very tight limits on anisotropic Hubble expansion and on shear and rotation (vorticity) in the Universe. We also know that the geometry of the Universe is very near to the idealized Robertson-Walker metric with only small perturbations which must be on the same scale as the CMB anisotropies. In 1968 Ehlers, Geren, and Sachs proved the theorem: If a family of freely-falling observers measure self-gravitating background radiation to be exactly isotropic, then the Universe is exactly Friedmann-Lemaitre-Robertson-Walker. This has been interpreted/extrapolated by me and others that the observed high degree of observed CMB isotropy and the assumption that our location is not special imply that the Universe's metric is nearly Robertson-Walker with perturbations of the order of $10^{-5}$. I have been quoting that result and encouraging the extension of the theorem. Now Stoeger, Maartens, and Ellis (1994) have shown that if all fundamental observers measure the cosmic microwave background radiation to be almost isotropic in an expanding Universe, then that Universe is almost spatially homogeneous and isotropic. This puts us on firmer footing and allows us to use the CMB isotropy ob- 


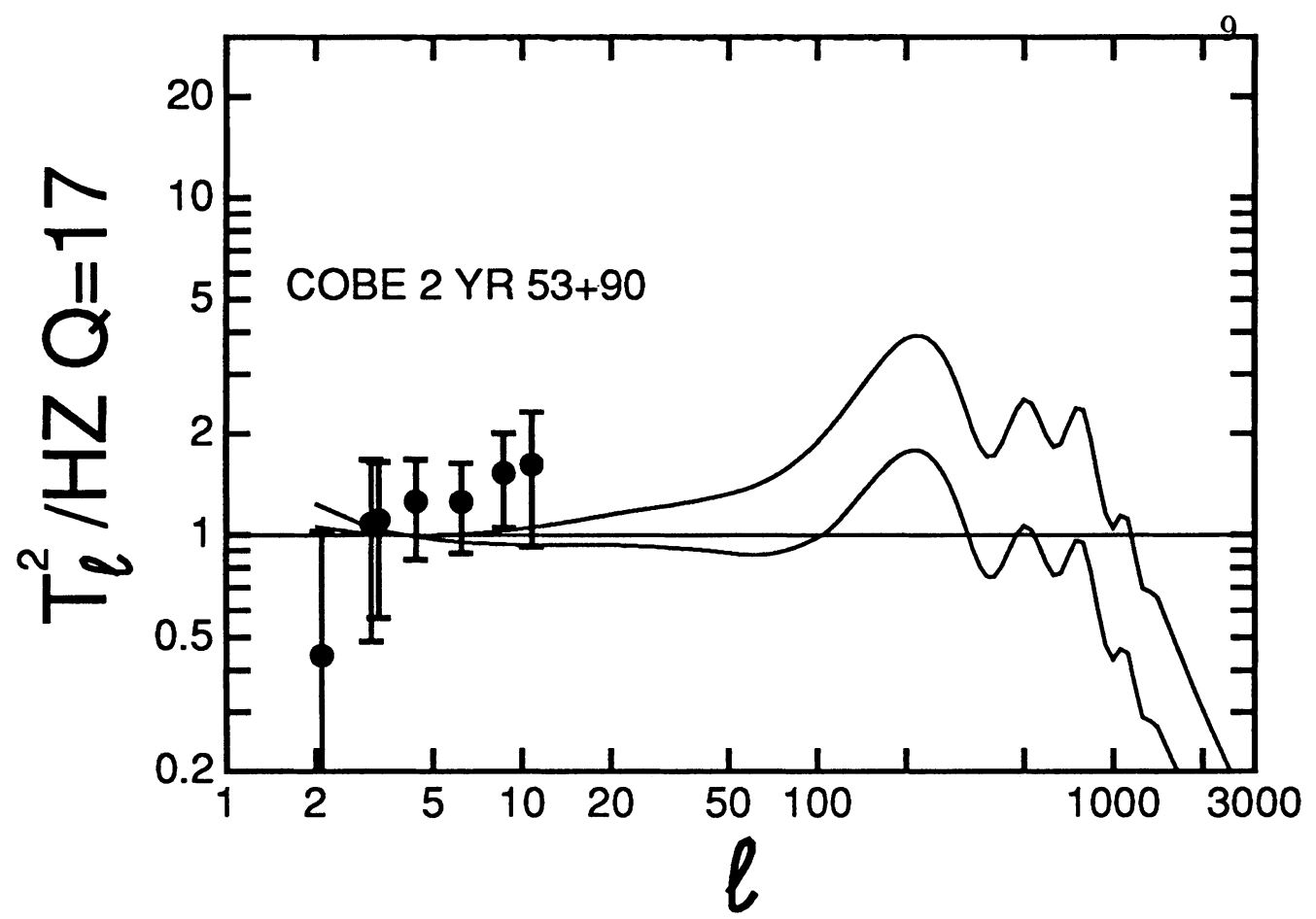

Figure 3. COBE DMR two-year data power spectrum: The horizontal line indicates the anisotropy level for a primordial scale-invariant model with rms quadrupole amplitude of $17 \mu \mathrm{K}$. The other two lines show the expected anisotropies with a scale-invariant and a tilted primordial spectrum as processed in a standard CDM model.

servations to set limits on the anisotropy, homogeneity, and dynamics of the Universe.

The observation of anisotropies opens one new cosmological test: probing the topology of the Universe. The simplest topology that we can imagine is an essentially isotropic and homogeneous Universe that is simply connected. However, we know of no constraints that actually require that the Universe be simply connected. It might in fact have the topology of a donut or many other possible objects. In recent times there have been reports of periodicity in the Universe both in terms of large scale structure of galaxies ( 128 Mpc) and quasars (much larger scale). This has led a number of people to suggest that the Universe is small with opposite faces identified (or some other combination). Such universes would be periodic on the identified axes and thus could not have anisotropies with wavelengths longer than their symmetry axes. The existence of very large angular scale anisotropies, i.e. the quadrupole, octopole, and hexadecapole, put stringent limits on the size of the Universe. Various analyses of the DMR data set a limit on the smallness of the Universe at about 0.5 of the Hubble diameter (e.g. Jing \& Fang 1994, and Costa \& Smoot 1994). This is an illustration 
of the power of the CMB as a cosmological probe.

However, the main work of the field is to investigate the origin of the primordial perturbations and their tie to large scale structure. The best way to do this will be to combine the DMR data with the data from other experiments in an effort to map out the full sky and power spectrum.

\section{Other Anisotropy Results}

Since the DMR announced the discovery of anisotropy, nine groups have reported CMB anisotropies. Figure 4 shows the current status of the CMB anisotropy power spectrum observations.

The 'MIT' FIRS experiment (Meyer et al. 1991, Page et al. 1990) is the only other experiment to map a significant portion of the sky. The FIRS experiment has $\mathrm{a} \sim 3^{\circ}$ beam width and covered nearly a quarter of the sky with a single balloon flight. The FIRS data correlate well with the DMR data (Ganga et al. 1993) and show a similar power spectrum (Ganga et al. 1994) consistent with scale invariance.

Tenerife (Watson et al. 1991) is also a large angular scale experiment (beam width $5^{\circ}$ ) that covers a differenced $\left(8^{\circ}\right)$ strip scanned on the sky by the earth's rotation. The Tenerife experiment has pointed out bumps on the sky as specific locations of anisotropy (Hancock et al. 1994). The ULISSE experiment (de Bernardis et al. 1992) reported upper limits on $6^{\circ}$ CMB anisotropy using balloon-borne bolometric observations. The Advanced Cosmic Microwave Explorer (ACME South Pole) (Gaier et al. 1992, Schuster et al. 1993) reported upper limits and detections of fluctuations operating with HEMT amplifiers. The Saskatoon "SK93" experiment (Wollack et al. 1993) used HEMT amplifiers to detect CMB anisotropy from Saskatoon, SK, Canada. Fluctuations were reported from South Pole observations by the Python experiment (Dragovan et al. 1993). The ARGO balloon-borne experiment (de Bernardis et al. 1994) observed a statistically significant signal with a $52^{\prime}$ beam. The Italian Antarctic Base (IAB) experiment (Piccirillo \& Calisse 1993) used bolometric techniques with a 50 ' Gaussian beam and reports anisotropy. The Millimeter-wave Anisotropy eXperiment (MAX) is a balloon-borne bolometric instrument with high sensitivity in the medium angular scale that has completed four flights detecting significant CMB fluctuations (Alsop et al. 1992, Meinhold et al. 1993, Devlin et al. 1994, Clapp et al. 1994). The Medium Scale Anisotropy Measurement (MSAM) balloon-borne experiment (Cheng et al. 1994) is a very similar balloon-borne medium-scale CMB anisotropy instrument but with a different chopping scheme that allows the results to be reported either as a difference or a triple difference, providing two effective window functions. Also from the South Pole the White Dish (WD) experiment (Tucker 


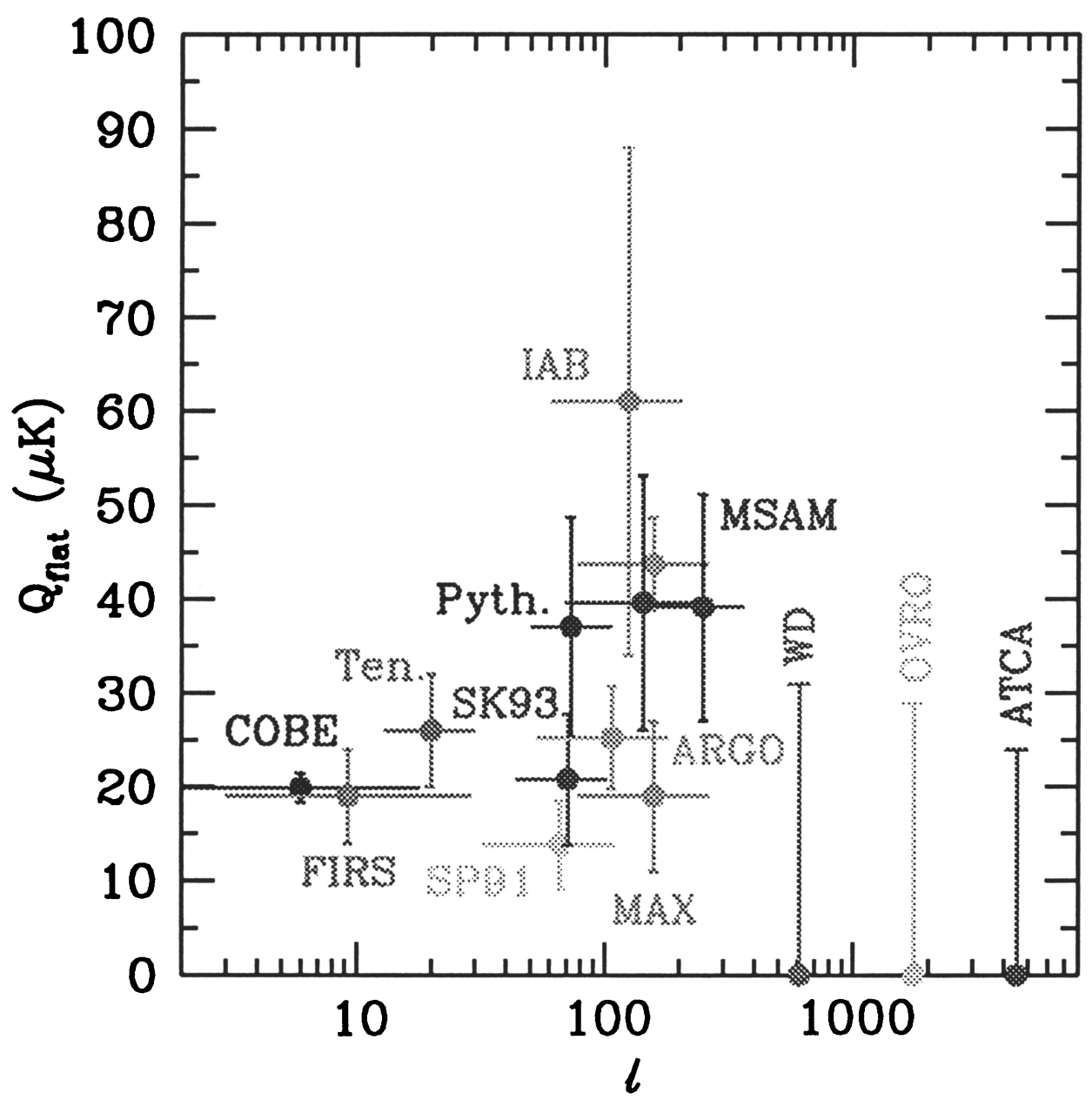

Figure 4. Current status of CMB anisotropy power spectrum observations adapted from Scott and White 1994. The amplitudes plotted are the quadrupole amplitudes for a flat (unprocessed scale-invariant spectrum of primordial perturbations, i.e. the horizontal line in Figure 3) anisotropy spectrum that would give the observed results for the experiment. Figure 3 gives an indication of the expected spectrum for a processed spectrum in a CDM model for comparison. 
et al. 1993) reports an upper limit on CMB anisotropy. Arc-minute scale anisotropy limits came from the Owens Valley Radio Observatory (OVRO) (Myers et al. 1993). The Australia Telescope Compact Array (ATCA) was used to place upper limits on CMB anisotropy in a synthesized image (Subrahmayan et al. 1993).

It appears that the reported results, though scattered, are actually in rough agreement with each other and with many models. The current goal moment is to refine the results and our ability to distinguish between models. A hot topic is whether the data show evidence for a Doppler peak and if that peak is in the right location. The existence and location of a peak would go far in telling us not only whether we are on the right track with these models, and if there is a connection with large scale structure formation, but also about various cosmological parameters.

\section{Interpretation, Future}

In two short years the field of CMB anisotropy observations and theory has made great strides. Until April 1992 all plots of CMB anisotropy showed only upper limits, except for the $\ell=1$ dipole. Now we are beginning to trace out the shape of the power spectrum and to make maps of the anisotropies. This promises to deliver a wealth of new information to cosmology and to connect to other fields. Already we are seeing plots showing the CMB anisotropy spectrum related to and overlaid on the primordial density perturbation power spectrum and attempts to reconstruct the inflaton potential. These are the first new steps in a new period of growth.

The COBE DMR has now released the first two years of its data and the full four-year data set is being processed and prepared for release in 1995. We can expect improved results from the DMR on the large angular scales but the scientific interest has moved to covering the full spectrum and learning what the medium and small angular scales will tell us. Already new experiments are underway. Nearly every group has new data under analysis and is also at work on developing new experiments. The first of these are the natural extensions of the ongoing experiments. Some groups are considering novel approaches. Real long-term progress depends on avoiding potential foregrounds: fluctuations of the atmosphere emission, a source of noise that that largely overwhelms recent advances in detector technology, and Galactic and extragalactic signals. This requires sufficient information and observing frequencies to separate the various components. It also means going above the varying atmosphere. Collaborations are working on longduration ballooning instruments. Ultimately, as COBE has shown, going to space really allows one to overcome the atmospheric effects and to get data in a very stable and shielded environment. A number of groups are 
working on designs for new satellite experiments. The COBRAS/SAMBA mission (Mandolesi et al. 1994) leads the way in the multi-wavelength and benign orbit location. With the new data that are appearing and can be expected and ultimately with the COBRAS/SAMBA mission we can look forward to a very significant improvement in our knowledge of cosmology.

\section{Acknowledgements}

Thanks to Eric Gawiser and Alan Kogut for reading and reviewing this paper. We acknowledge the excellent work of those contributing to the COBE-DMR. COBE is supported by the office of Space Sciences of NASA Headquarters. Goddard Space Flight Center (GSFC), with the scientific guidance of the COBE Science Working Group, is responsible for the development and operation of COBE. This work is supported in part by the Director, Office of Energy Research, Office of High Energy and Nuclear Physics, Division of High Energy Physics of the U.S. Department of Energy under Contract No. DE-AC03-76SF00098.

\section{References}

Alsop, D. C., et al. 1992, ApJ, 395, 317

Bennett, C.L., et al. 1992, ApJ, 396, 5.

Bennett, C.L., et al. 1994 ApJ, 436, 423.

de Bernardis, P., et al., 1992, ApJ, 396, L57

de Bernardis, P., et al. 1994, ApJ, 422, L33

Cayon, L., \& Smoot, G.F., 1994 submitted to ApJ

Cheng, E.S., et al., 1994, ApJ, 422, L37

Clapp, A.C., et al., 1994, ApJL, 433, L57

Costa, A,O., \& Smoot, G.F., submitted to ApJ

Devlin, M.J., et al., 1994, ApJL, 430, L1

Dragovan, M., et al., 1993, ApJ, 427, L67-70

Ehlers, J., Geren, P., Sachs, R.K., J. Math. Phys., 9, 1344.

Gaier, T., et al. 1992, ApJ, 398, L1

Ganga, K., Page, L., Cheng, E., \& Meyer, S. 1993, ApJ., 410, L57.

Ganga, K., Page, L., Cheng, E., \& Meyer, S. 1994, ApJ, 432, L15

Górski, K. M., Hinshaw, G., Banday, A. J., Bennett, C. L., Wright, E. L., Kogut, A., Smoot, G. F. \& Lubin, P. 1994, ApJ, 430, L85

Gush, H., Halpern, M., \& Winshow, E., 1990, Phys. Rev, Lett., 65, 537.

Hancock, S., et al., 1994, Nature, 367, 333

Harrison, E.R., 1970, Phys. Rev. D1, 2726

Jing, Y.P., \& Fang, L.Z., 1994, PRL, 73 (14), 1882

Kogut et al., 1992, ApJ, 401, 1.

Lineweaver, C.L., Hancock, S., et al. 1994 submitted to ApJ

Mandolesi, N., et al. 1994 accepted Planetary and Space Sciences. 
Mather, J. C., et al. 1994, ApJL, 420, 439-44

Meinhold, P., \& Lubin, P. 1991, ApJ, 370, L11

Meinhold, P., et al. 1993, ApJ, 409, L1

Meyer S. S., Page, L., \& Cheng, E. S. 1991, ApJ, 371, L7

Myers, S. T., Readhead, A. C. S. \& Lawrence, C. R. 1993, ApJ, 405, 8

Penzias, A.A., \& Wilson, R.W. 1965, ApJ, 142, 419

Piccirillo. L., and Calisse, O. ApJ, 411, 529-533.

Sachs, R.K., \& Wolfe, A.M., 1967, ApJ, 147, 73

Scott, D., \& White, M., 1994, CWRU Workshop Proceedings.

Smoot, G.F., et al., 1992, Ap.J. 396, L1.

Smoot, G.F., et al., 1994, Ap.J. 437, 1.

Subrahmayan, R., Ekers, R., Sinclair, M. \& Silk, J. 1993, MNRAS, 263, 416

Stoeger, W., Maartens, R., \& Ellis, G.F.R., ApJ in press

Tucker, G.S., Griffin, G., Nguyen, H. \& Peterson, J.B. 1993, ApJ, 419, L45

Watson, R.A. et al., 1992 Nature 357, 660

Wollack, E. J., et al., 1993, ApJ, 419, L49

Wright, E.L., et al., 1992, ApJ, 396, L13.

Wright, E.L., Smoot, G.F., Bennett, C., \& Lubin, P., 1994, ApJ, 436, 443

Zel'dovich, Ya.B., 1972 Mon. Not. R. astr. Soc. 160, 1. 